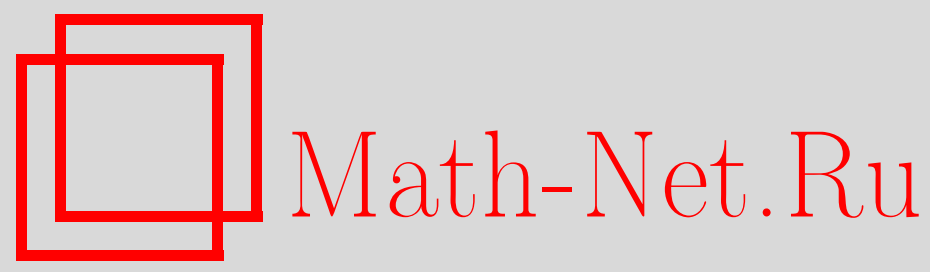

Б. С. Кашин, Т. Ю. Куликова, Замечание об описании фреймов общего вида, Матем. заметки, 2002, том 72, выпуск $6,941-945$

DOI: https://doi.org/10.4213/mzm675

Использование Общероссийского математического портала Math-Net.Ru подразумевает, что вы прочитали и согласны с пользовательским соглашением http://www . mathnet.ru/rus/agreement

Параметры загрузки:

IP: 52.23 .180 .231

26 апреля 2023 г., 16:07:32

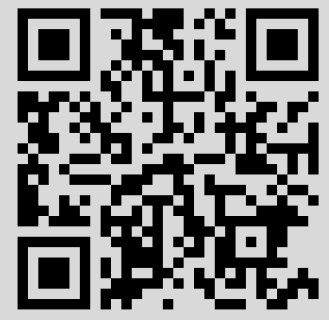




\section{ЗАМЕЧАНИЕ ОБ ОПИСАНИИ ФРЕЙМОВ ОБЩЕГО ВИДА}

\section{Б. С. Кашин, Т. Ю. Куликова}

Пусть $H$ - гильбертово пространство. Система ненулевых элементов $\Phi=\left\{\varphi_{j}: j \in \mathbb{N}\right\} \subset H$ назьвается фреймом, если выполнены неравенства

$$
A\|g\|_{H}^{2} \leqslant \sum_{j=1}^{\infty}\left|\left(g, \varphi_{j}\right)\right|^{2} \leqslant B\|g\|_{H}^{2} \quad \forall g \in H
$$

где $0<A \leqslant B<\infty$ - абсолютные постоянные, а $\|\cdot\|$ и $(\cdot, \cdot)$ - норма и скалярное произведение в $H$. Постоянные $B$ и $A$ назьваются соответственно верхней и нижней әраничами фрейма $\Phi$, а их отношение $\kappa=B / A$ называется коэффициентом обусловленности и обозначается $\kappa(\Phi)$. В случае, когда $\kappa(\Phi)=1$, т.е. $A=B$, фрейм $\Phi$ называется жестким. Фреймы были введены в 1952 году в работе Дафффина и Шеффера [1] (см. также [2]), однако некоторые результаты о фреймах содержались в неявном виде и в более ранних работах. В частности, следующий результат известен в квантовой теории информации и некоторых областях функционального анализа как теорема Наймарка и датируется 1940 годом.

Tеорема А (см. [3, гл. 10], [4], [9]). Для любого жесткого фрейма $\Phi=\left\{\varphi_{j}: j \in \mathbb{N}\right\}$ в гильбертовом пространстве $H$ найдутся гильбертово пространство $H^{\prime} \supset$ и ортонормированный базис $\Psi=\left\{\psi_{j}: j \in \mathbb{N}\right\}$ пространства $H^{\prime}$ такие, что

$$
\varphi_{j}=\pi_{H^{\prime} \rightarrow H}\left(\psi_{j}\right), \quad j=1,2, \ldots,
$$

əде $\pi_{H^{\prime} \rightarrow H}$ - оператор ортогонального проектирования из $H^{\prime}$ в $H$.

Близкие к теореме А результаты содержатся также в работе Козлова 1948 года, где, по существу, исследовались жесткие фреймы (см. [5], а также [6] и [7, теорема 8.3]). Недавно результаты смежные с теоремой А были получены Лукашенко [8].

В последние годы фреймы нашли разнообразные приложения в прикладной математике, в частности, при построении алгоритмов сжатия изображений. В этой связи представляется естественньпм дать описание фреймов общего вида, аналогичное теореме А. Мы не нашли соответствующего резултата в литературе и устанавливаем его в этой заметке. Приводимое ниже доказательство с нашей точки зрения проще, чем известные доказательства теоремы Наймарка (рассуждения ниже, в предположении, что $\kappa(\Phi)=1$, аналогичны проверке элементарного факта из линейной алгебры о возможности дополнения ортонормированного набора векторов $\left\{v_{i}, i=1,2, \ldots, s\right\} \subset \mathbb{R}^{n}$, $1 \leqslant s<n$, до ортонормированного базиса в $\mathbb{R}^{n} ;$ весьма прозрачен конечномерный вариант установленной ниже теоремы 1 и в случае, когда $\kappa(\Phi)>1)$.

Прежде, чем формулировать и доказьвать наш результат, введем некоторые обозначения. Пусть $\Lambda$ - некоторое счетное множество с заданным на нем порядком (иными словами, мы считаем, что фиксировано взаимно однозначное отображение $\Lambda$ на натуральный ряд $\mathbb{N}$ ). Через $\ell^{2}(\Lambda)$ обозначим гильбертово пространство наборов чисел $\{c \omega, \omega \in \Lambda\}$, занумерованных элементами из $\Lambda$ с нормой

$$
\left(\sum_{\omega \in \Lambda}\left|c_{\omega}\right|^{2}\right)^{1 / 2}
$$

Классическое гильбертово пространство $\ell^{2}=\ell^{2}(\mathbb{N})$ соответствует случаю, когда $\Lambda=\mathbb{N}$ и на $\Lambda$ задан естественный порядок. В этом случае для $j$-го элемента $a_{j}$ последовательности $a=\left\{a_{j}\right.$ : $j \in \mathbb{N}\} \in \ell^{2}$ используем также обозначение $(a)_{j}$. Система элементов $\Psi=\left\{\psi_{\omega}: \omega \in \Lambda\right\}$ гильертова пространства $H^{\prime}$ называется базисом Pисса в $H^{\prime}$, если $\Psi$ полна в $H^{\prime}$ и для любого набора чисел $\left\{c_{\omega}: \omega \in \Lambda\right\} \in \ell^{2}(\Lambda)$ выполняются оценки

$$
A^{1 / 2}\left(\sum_{\omega \in \Lambda}\left|c_{\omega}\right|^{2}\right)^{1 / 2} \leqslant\left\|\sum_{\omega \in \Lambda} c_{\omega} \psi_{\omega}\right\|_{H^{\prime}} \leqslant B^{1 / 2}\left(\sum_{\omega \in \Lambda}\left|c_{\omega}\right|^{2}\right)^{1 / 2}
$$


где $0<A \leqslant B<\infty$ - абсолютные постоянные (о базисах Рисса см., например, [7, с. 17]; обычно $\Lambda=\mathbb{N}$; отметим еще, что свойство системы быть базисом Рисса не зависит от порядка элементов этой системы).

Теорема 1. Для того чтобы система әлементов $\Phi=\left\{\varphi_{j}: j \in \mathbb{N}\right\}$ зильбертова пространства $H$ являлась фреймом с границами $A$ и $B, A \leqslant B$, необходимо и достаточно, чтобы нашлось гильбертово пространство $H^{\prime}$, содержащее $H$, и базис Рисса $\Psi=\left\{\psi_{j}: j \in \mathbb{N}\right\}$ в $H^{\prime}$ со свойством (2) $($ при $\Lambda=\mathbb{N})$ такие, ито

$$
\varphi_{j}=\pi_{H^{\prime} \rightarrow H}\left(\psi_{j}\right), \quad j=1,2, \ldots
$$

Ниже для определенности считаем, что $H$ - действительное гильбертово пространство. Следующий факт, по существу, хорошо известен.

УтвЕРЖДЕнИЕ 1. Пусть $\Lambda$ - некоторое счетное множество $u V=\left\{v_{\omega}: \omega \in \Lambda\right\}-$ базис Рисса в гильбертовом пространстве $H$, причем для всех $с \in \ell^{2}(\Lambda)$

$$
A^{1 / 2}\left(\sum_{\omega \in \Lambda} c_{\omega}^{2}\right)^{1 / 2} \leqslant\left\|\sum_{\omega \in \Lambda} c_{\omega} v_{\omega}\right\|_{H} \leqslant B^{1 / 2}\left(\sum_{\omega \in \Lambda} c_{\omega}^{2}\right)^{1 / 2},
$$

где $A>0, B$ - абсолютные постоянные. Тогда сопряженная ж $V$ система

$$
V^{*}=\left\{v_{\mu}^{*}: \mu \in \Lambda\right\}
$$

является базисом Рисса в сопряженном пространстве $H^{*}$ и выполняется оченка

$$
\frac{1}{B^{1 / 2}}\left(\sum_{\mu \in \Lambda} \beta_{\mu}^{2}\right)^{1 / 2} \leqslant\left\|\sum_{\mu \in \Lambda} \beta_{\mu} v_{\mu}^{*}\right\|_{H^{*}} \leqslant \frac{1}{A^{1 / 2}}\left(\sum_{\mu \in \Lambda} \beta_{\mu}^{2}\right)^{1 / 2} .
$$

(Напомним, что сопряженная система однозначно определяется соотношениями

$$
\left\langle v_{\mu}^{*}, v_{\omega}\right\rangle= \begin{cases}1, & \text { если } \mu=\omega \\ 0, & \text { если } \mu \neq \omega\end{cases}
$$

где $\langle f, g\rangle$ - значение функционала $f \in H^{*}$ на элементе $g \in H$.)

ДоКАЗАТЕЛЬСтво. Полнота и минимальность системы $V^{*}$ вытекает из общих результатов о базисах (см. [7, гл. 1]). Проверим справедливость неравенств (4). Имеем

$$
\begin{aligned}
\left\|\sum_{\mu \in \Lambda} \beta_{\mu} v_{\mu}^{*}\right\|_{H^{*}} & =\sup _{\left\{c_{\omega}\right\}:\left\|\sum_{\omega \in \Lambda} c_{\omega} v_{\omega}\right\|_{H} \leqslant 1}\left\langle\sum_{\mu \in \Lambda} \beta_{\mu} v_{\mu}^{*}, \sum_{\omega \in \Lambda} c_{\omega} v_{\omega}\right\rangle \\
& =\sup _{\left\{c_{\omega}\right\}:\left\|\sum_{\omega \in \Lambda} c_{\omega} v_{\omega}\right\|_{H} \leqslant 1} \sum_{\omega \in \Lambda} \beta_{\omega} c_{\omega} \geqslant \frac{1}{B^{1 / 2}}\left(\sum_{\omega \in \Lambda} \beta_{\omega}^{2}\right)^{1 / 2},
\end{aligned}
$$

так как в силу (3) имеет место включение

$$
\left\{\left\{c_{\omega}\right\}:\left\|\sum c_{\omega} v_{\omega}\right\|_{H} \leqslant 1\right\} \supset\left\{\left\{c_{\omega}\right\}:\left(\sum_{\omega \in \Lambda} c_{\omega}^{2}\right)^{1 / 2} \leqslant \frac{1}{B^{1 / 2}}\right\} .
$$

Аналогично,

$$
\left\|\sum_{\mu \in \Lambda} \beta_{\mu} v_{\mu}^{*}\right\|_{H^{*}}=\sup _{\left\{c_{\omega}\right\}:\left\|\sum_{\omega \in \Lambda} c_{\omega} v_{\omega}\right\|_{H} \leqslant 1} \sum_{\omega \in \Lambda} \beta_{\omega} c_{\omega}
$$


и в силу включения

$$
\left\{\left\{c_{\omega}\right\}:\left\|\sum_{\omega \in \Lambda} c_{\omega} v_{\omega}\right\|_{H} \leqslant 1\right\} \subset\left\{\left\{c_{\omega}\right\}:\left(\sum_{\omega \in \Lambda} c_{\omega}^{2}\right)^{1 / 2} \leqslant \frac{1}{A^{1 / 2}}\right\}
$$

мы имеем

$$
\left\|\sum_{\mu \in \Lambda} \beta_{\mu} v_{\mu}^{*}\right\|_{H^{*}} \leqslant \frac{1}{A^{1 / 2}}\left(\sum_{\mu \in \Lambda} \beta_{\mu}^{2}\right)^{1 / 2}
$$

Утверждение 1 доказано.

Из утверждения 1 вытекает, что для любого базиса Рисса $V=\left\{v_{\omega}: \omega \in \Lambda\right\}$ со свойством (3) и для любого элемента $f \in H^{*}$ выполняются соотношения

$$
A\|f\|_{H^{*}}^{2} \leqslant \sum_{\omega \in \Lambda}\left\langle f, v_{\omega}\right\rangle^{2} \leqslant B\|f\|_{H^{*}}^{2} .
$$

Действительно, применяя утверждение 1 для разложения функционала $f$ по базису $\left\{v_{\mu}^{*}: \mu \in \Lambda\right\}$ :

$$
f=\sum_{\omega \in \Lambda}\left\langle f, v_{\omega}\right\rangle v_{\omega}^{*}
$$

мы получаем (5). Используя изометрию пространств $H$ и $H^{*}$, мы из (5) вьводим, что в условиях утверждения 1 для любого $g \in H$ вьполнено

$$
A\|g\|_{H}^{2} \leqslant \sum_{\omega \in \Lambda}\left(g, v_{\omega}\right)^{2} \leqslant B\|g\|_{H}^{2} .
$$

ДоКАЗАТЕЛЬСтво теоРемы 1. Достаточность. Пусть $\Psi=\left\{\psi_{j}: j \in \mathbb{N}\right\}$ - базис Рисса в $H^{\prime}$, $H^{\prime} \supset H$, и выполнены соотношения (2) при $\Lambda=\mathbb{N}$. Пусть также $\varphi_{j}=\pi_{H^{\prime} \rightarrow H}\left(\psi_{j}\right), j=1,2, \ldots$ Тогда для любого элемента $g \in H$ выполнено

$$
\left(g, \varphi_{j}\right)=\left(g, \psi_{j}\right), \quad j=1,2, \ldots,
$$

a, значит, для любого $g \in H$

$$
A\|g\|_{H}^{2} \leqslant \sum_{j=1}^{\infty}\left(g, \varphi_{j}\right)^{2} \leqslant B\|g\|_{H}^{2}
$$

т.е. система $\left\{\varphi_{j}: j \in \mathbb{N}\right\}-$ фрейм.

Необходимость. Не ограничивая общности, считаем, что $H=\ell^{2}$. Образуем бесконечную матрицу $R, j$-й столбец которой совпадает с последовательностью $\varphi_{j} \in \ell^{2}, j=1,2, \ldots$ Пусть $v_{i}$, $i=1,2, \ldots,-$ строки матрицы $R$. Тогда неравенства (1) означают, что для любой последовательности $\alpha=\left\{\alpha_{i}: i=1,2, \ldots\right\} \in \ell^{2}$ выполнены оценки

$$
A\|\alpha\|_{\ell^{2}}^{2} \leqslant\left\|\sum_{i=1}^{\infty} \alpha_{i} v_{i}\right\|_{\ell^{2}} \leqslant B\|\alpha\|_{\ell^{2}}^{2}
$$

Пусть $L$ - замыкание (по норме $\ell^{2}$ ) линейной оболочки системы $V=\left\{v_{i}: i \in \mathbb{N}\right\}$. В силу (7) $\operatorname{dim} L=\infty$ и $V$ - базис Рисса в $L$. Рассмотрим ортогональное дополнение $L^{\perp}$ к $L$ в $\ell^{2}$. Пусть $W=\left\{w_{\nu}: \nu \in M\right\}-$ произвольный ортогональный базис в $L^{\perp}$, причем

$$
\forall \nu \in M \quad A \leqslant\left\|w_{\nu}\right\|_{\ell^{2}}^{2} \leqslant B
$$


где $M$ - некоторое счетное, конечное или пустое (в зависимости от размерности подпространства $\left.L^{\perp}\right)$ множество индексов, $M \cap \mathbb{N}=\varnothing$ и элементы системы $W$ "занумерованы" точками из $M$. Пусть $\Lambda=\mathbb{N} \cup M$ и система $\Gamma=\left\{\gamma_{\omega}: \omega \in \Lambda\right\} \subset \ell^{2}$ задается соотношениями

$$
\gamma_{\omega}= \begin{cases}v_{i} & \text { если } \omega=i \in \mathbb{N}, \\ w_{\nu} & \text { если } \omega=\nu \in M .\end{cases}
$$

Тогда легко видеть, что $\Gamma$ - базис Рисса в $\ell^{2}$, причем

$$
\left(A \sum_{\omega \in \Lambda} c_{\omega}^{2}\right)^{1 / 2} \leqslant\left\|\sum_{\omega \in \Lambda} c_{\omega} \gamma_{\omega}\right\|_{\ell^{2}} \leqslant\left(B \sum_{\omega \in \Lambda} c_{\omega}^{2}\right)^{1 / 2} .
$$

Действительно, для любого элемента $g \in \ell^{2}$ имеет место разложение

$$
g=g_{L}+g_{L \perp}
$$

где

Если

$$
g_{L}=\pi_{\ell^{2} \rightarrow L}(g), \quad g_{L^{\perp}}=\pi_{\ell^{2} \rightarrow L^{\perp}}(g) .
$$

$$
g_{L}=\sum_{i \in \mathbb{N}} \alpha_{i} v_{i}, \quad g_{L^{\perp}}=\sum_{\nu \in M} \beta_{\nu} w_{\nu}
$$

то в силу ортогональности $g_{L}$ и $g_{L}$, , а также неравенств (7) и (8)

$$
\begin{aligned}
& \|g\|^{2}=\left\|g_{L}\right\|_{\ell^{2}}^{2}+\left\|g_{L^{\perp}}\right\|_{\ell^{2}}^{2} \leqslant B \sum_{i \in \mathbb{N}} \alpha_{i}^{2}+\sum_{\nu \in M} \beta_{\nu}^{2}\left\|w_{\nu}\right\|_{\ell^{2}}^{2} \leqslant B\left(\sum_{i \in \mathbb{N}} \alpha_{i}^{2}+\sum_{\nu \in M} \beta_{\nu}^{2}\right), \\
& \|g\|^{2}=\left\|g_{L}\right\|_{\ell^{2}}^{2}+\left\|g_{L^{\perp}}\right\|_{\ell^{2}}^{2} \geqslant A \sum_{i \in \mathbb{N}} \alpha_{i}^{2}+\sum_{\nu \in M} \beta_{\nu}^{2}\left\|w_{\nu}\right\|_{\ell^{2}}^{2} \geqslant A\left(\sum_{i \in \mathbb{N}} \alpha_{i}^{2}+\sum_{\nu \in M} \beta_{\nu}^{2}\right),
\end{aligned}
$$

что доказывает (10).

Заффиксировав произвольный порядок на множестве $\Lambda$, рассмотрим "матрицу"

$$
\widetilde{R}=\left\{r_{\omega, j}: \omega \in \Lambda, j \in \mathbb{N}\right\},
$$

где для всех $\omega \in \Lambda$ и $j \in \mathbb{N}$

$$
r_{\omega, j}= \begin{cases}\left(v_{\omega}\right)_{j}, & \text { если } \omega \in \mathbb{N}, \\ \left(w_{\omega}\right)_{j}, & \text { если } \omega \in M .\end{cases}
$$

Пусть $\psi_{j}, j=1,2, \ldots,-$ “ “-й столбец матрицы $\widetilde{R}$ ”, т.е. набор чисел $\left\{r_{\omega, j}: \omega \in \Lambda\right\} \subset \ell^{2}(\Lambda)$. Пусть

$$
\pi_{0}=\pi_{\ell^{2}(\Lambda) \rightarrow \ell^{2}(\mathbb{N})}
$$

- оператор ортогонального проектирования из гильбертова пространства $\ell^{2}(\Lambda)$ на его подпространство $\ell^{2}(\mathbb{N})$. Тогда ясно, что $\pi_{0}\left(\psi_{j}\right)=\varphi_{j}, j=1,2, \ldots$, и нам остается проверить, что система $\left\{\psi_{j}\right\}$ - базис Рисса в $H^{\prime}=\ell^{2}(\Lambda)$ и справедливы неравенства (2). Неравенства (10) означают, что для любого элемента $c=\left\{c_{\omega}\right\} \in \ell^{2}(\Lambda)$ выполнено

$$
A\|c\|_{\ell^{2}(\Lambda)}^{2} \leqslant \sum_{j=1}^{\infty}\left(c, \psi_{j}\right)^{2} \leqslant B\|c\|_{\ell^{2}(\Lambda)}^{2} .
$$

Из (11) вытекает полнота системы $\left\{\psi_{j}\right\}$ в $\ell^{2}(\Lambda)$. Далее, для любой последовательности $\rho=\left\{\rho_{j}\right\} \in$ $\ell^{2}(\mathbb{N})$ рассмотрим ряд в $\ell^{2}(\Lambda)$ :

$$
\sum_{j=1}^{\infty} \rho_{j} \psi_{j}
$$


Тогда (см. (9))

$$
\left\|\sum_{j=1}^{\infty} \rho_{j} \psi_{j}\right\|_{\ell^{2}(\Lambda)}^{2}=\sum_{\omega \in \Lambda}\left(\rho, \gamma_{\omega}\right)^{2}
$$

Так как $\Gamma=\left\{\gamma_{\omega}\right\}$ - базис Рисса в $\ell^{2}$ и имеет место (10), то мы можем воспользоваться соотношением (6) и, учитьвая (12), получить, что

$$
A \sum_{j=1}^{\infty} \rho_{j}^{2} \leqslant\left\|\sum_{j=1}^{\infty} \rho_{j} \psi_{j}\right\|_{\ell^{2}(\Lambda)}^{2} \leqslant B \sum_{j=1}^{\infty} \rho_{j}^{2}
$$

Теорема 1 доказана.

В заключение, пользуясь случаем, сделаем дополнение к теореме 1 нашей недавней заметки [10]. Аналогично [10] могут оцениваться снизу величины

$$
\gamma_{n}(W, \Phi, D)=\sup _{f \in W \Lambda_{n},\left\{c_{j}\right\}_{j \in \Lambda_{n}}:\left(\sum c_{j}^{2}\right)^{1 / 2} \leqslant D\|f\|}\left\|f-\sum_{j \in \Lambda_{n}} c_{j} \varphi_{j}\right\|_{H},
$$

где $W \subset H, \Phi=\left\{\varphi_{j}\right\}-\not р$ рейм в $H$, а $\Lambda_{n}$ пробегает семейство всех $n$-элементньг подмножеств $\mathbb{N}$.

\section{СПИСОК ЦИТИРОВАННОЙ ЛИТЕРАТУРЫ}

1. Duffin R., Schaeffer A. // Trans. Amer. Math. Soc. 1952. V. 72. № 2. P. 341-266. 2. Добеши И. Десять лекций по вейвлетам. М.-Ижевск: РХД, 2001. 3. Ахиезер Н. И., Глазман И. М. Теория линейных операторов в гильбертовом пространстве. М.: Наука, 1966. 4. Наймарк М. А. // Изв. АН СССР. Сер. матем. 1940. Т. 4. № 3. С. 277-318. 5. Козлов В. Я. // Матем. сб. 1948. Т. 23. № 3. С. 441-474. 6. Олевский А. М. // Матем. заметки. 1969. Т. 6. С. 737-747. 7. Кашин Б.С., Саакян А. А. Ортогональные ряды. 2-е изд. М.: АФЦ, 1999. 8. Лукашенко Т. П. // Матем. сб. 1997. Т. 188. №12. С. 57-72. 9. Холево А. С. Введение в квантовую теорию информации. М.: МЦНМО, 2002. 10. Кашин Б. С., Куликова Т. Ю. // Матем. заметки. 2002. T. 72 . № 2. C. 312-315.

(Б. С. Кашин) Математический институт им. В. А. Стеклова РАН (Т. Ю. Куликова) Научно-исследовательский актуарно-финансовьй центр 